\title{
Creme de leite UHT homogeneizado: perfil sensorial e sua relação com a expectativa de consumo
}

\author{
UHT homogenized cream: sensory profile and the relation \\ with consumption expectancy
}

Pedro Pio Campregher Augusto ${ }^{1 *}$ (D), Camila de Souza Paglarini ${ }^{1}$, lara Maria Cerqueira Magalhães ${ }^{1}$, Mayra Fernanda de Souza Campos' ${ }^{1}$, Rachel Bertoldo1, Tatiana Ferrari Baldini ${ }^{1}$, Helena Maria André Bolini ${ }^{1}$ (i)

${ }^{1}$ Universidade Estadual de Campinas (UNICAMP), Faculdade de Engenharia de Alimentos, Departamento de Alimentos e Nutrição, Campinas/SP - Brasil

${ }^{*}$ Corresponding Author: Pedro Pio Campregher Augusto, Universidade Estadual de Campinas (UNICAMP),

Faculdade de Engenharia de Alimentos, Departamento de Alimentos e Nutrição, Rua Monteiro Lobato, 80, Cidade Universitária Zeferino Vaz, CEP: 13083-862, Campinas/SP - Brasil, e-mail: pedrupio@gmail.com

Cite as: Augusto, P. P. C., Paglarini, C. S., Magalhães, I. M. C., Campos, M. F S., Bertoldo, R., Baldini, T. F., \& Bolini, H. M. A. (2020). UHT homogenized cream: sensory profile and the relation with consumption expectancy. Brazilian Journal of Food Technology, 23, e2019149. https://doi.org/10.1590/1981-6723.14919

\begin{abstract}
Resumo
Creme de leite homogeneizado UHT em embalagem longa vida é amplamente consumido no Brasil. Versões livres de lactose e com teor reduzido de gordura também estão disponíveis do mercado, seguindo necessidades especiais de consumidores e tendência de ingestão controlada de macronutrientes, respectivamente. Além de fatores como preço e disponibilidade, os consumidores selecionam os produtos com base em suas características sensoriais. Neste contexto, este estudo buscou avaliar como as características sensoriais do creme de leite UHT influenciam sua escolha e aceitação. Para isso, seis amostras comerciais de creme de leite UHT com teores de gordura variando de $17 \%$ a $20 \%$, sendo uma sem lactose, tiveram seus perfis sensoriais determinados por meio da Análise Descritiva Quantitativa (ADQ) e foram submetidos a testes de aceitabilidade e intenção de compra. Os dados obtidos foram correlacionados por meio da análise de regressão por mínimos quadrados parciais. Análises instrumentais de cor e reologia também foram realizadas. Descritores de textura tiveram maior influência sobre a aceitabilidade das amostras, sendo que as amostras percebidas pelos julgadores treinados como mais viscosas, encorpadas e consistentes obtiveram maiores médias no teste de aceitabilidade, ao contrário daquelas consideradas mais fluidas. Não foi verificada associação do teor de gordura com as propriedades reológicas das amostras, já que as duas amostras com $17 \%$ de gordura apresentaram valores instrumentais e sensoriais de viscosidade muito diferentes entre si. Uma delas foi considerada a mais fluida, e a outra a segunda mais viscosa, consistente e encorpada pelos julgadores treinados, o que foi confirmado pela análise instrumental.
\end{abstract}

Palavras-chave: Creme de leite UHT; Análise descritiva quantitativa; Direcionadores de preferência; Lácteos; Reduzido em gordura; Sem lactose. 


\begin{abstract}
UHT homogenized cream in long-life packaging is widely consumed in Brazil. Lactose free and low fat versions are also available in the market, following special consumer needs and controlled macronutrient intake trend, respectively. In addition to factors such as price and availability, consumers select products based on their sensory characteristics. In this context, this study aimed to evaluate how the sensory characteristics of UHT cream influence its choice and acceptance. To accomplish this, six commercial samples of UHT cream with fat content ranging from $17 \%$ to $20 \%$, one without lactose, had their sensory profiles determined by Quantitative Descriptive Analysis (QDA) and were submitted to acceptability and purchase intention tests. The data were correlated by Partial Least Squares Regression analysis. Instrumental analysis of color and rheology were also performed. Texture descriptors had a greater influence on the acceptability of the samples and the samples perceived by trained judges as more viscous, full-bodied and consistent obtained higher averages in the acceptability test, as opposed to those considered more fluid. There was no association between fat content and rheological properties of the samples, since the two samples with $17 \%$ fat presented very different instrumental and sensory viscosity values. One was considered the most fluid and the other the second most viscous, consistent and full-bodied by trained judges, which was confirmed by instrumental analysis.
\end{abstract}

Keywords: UHT cream; Quantitative descriptive analysis; Preference drivers; Dairy; Low fat; Lactose free.

\title{
1 Introdução
}

Creme de leite é a emulsão óleo em água concentrada obtida da separação física do leite cru, comumente utilizado como ingrediente alimentício devido a sua característica cremosidade, proporcionada pela fina dispersão de glóbulos de gordura protegidos por uma membrana emulsificante (Hoffmann, 2011a; Moura et al., 2001). Tradicionalmente, é considerado um ingrediente de consumo restrito, já que a gordura láctea corresponde a apenas $4 \%$ da composição do leite e exige armazenamento sob refrigeração, limitando sua disponibilidade (Hoffmann, 2011b). No entanto, a produção brasileira de todas as variantes de creme de leite teve um crescimento contínuo no período de 2010 a 2016, sendo que em 2015 a produção atingiu o máximo do período, 409 mil toneladas (Gomes et al., 2017). Além disso, cremes de leite com menor teor de gordura e submetidos a tratamentos que aumentam sua vida útil tornaram-se bastante acessíveis nos mercados brasileiros. O desenvolvimento de produtos adequados a dietas de ingestão controlada de calorias e gorduras é uma tendência importante no setor de lácteos brasileiro (Gallina \& Antunes, 2017).

A classificação do creme de leite não é uniforme ao redor do mundo, e a nomenclatura utilizada pode variar de acordo com o teor de gordura, com o uso pretendido ou com os tratamentos aos quais o produto é submetido (Deosarkar et al., 2016; Lee et al., 2018). No Brasil, a legislação vigente determina que, para ser nomeado creme de leite, o produto deve possuir no mínimo $10 \%$ de gordura láctea, e aqueles com teor de matéria gorda menor que 19,9\% são classificados como creme de leite leve (Brasil, 1996).

Os tratamentos de homogeneização e ultrapasteurização (UHT) do creme de leite têm como objetivo garantir sua estabilidade físico-química e microbiológica durante o tempo de vida de prateleira, respectivamente (Walstra et al., 2005). A homogeneização do leite e seus derivados, como o creme, é um processo mecânico no qual o tamanho dos glóbulos de gordura é reduzido, de modo a favorecer sua interação com a fase aquosa e diminuir o risco de coalescência, dificultando a separação de fase durante o armazenamento (Fox, 2011; Wilbey, 2011).

A oferta e demanda de produtos lácteos ultrapasteurizados UHT (ultra-high temperature) aumentou significativamente nos últimos anos devido à conveniência e praticidade conferida pela sua estabilidade à temperatura ambiente, que pode chegar até 12 meses. O tratamento UHT envolve aquecimento a temperaturas entre $135{ }^{\circ} \mathrm{C}$ e $150{ }^{\circ} \mathrm{C}$ por alguns segundos, que esterilizam o produto e destroem microrganismos patogênicos, bem como a maioria das suas formas esporuladas (Malmgren et al., 2017). 
Contudo, as altas temperaturas utilizadas na ultrapasteurização do creme de leite podem promover o escurecimento não enzimático, especialmente naqueles com teor reduzido de lactose, já que a hidrólise deste dissacarídeo aumenta a concentração de açúcares redutores que participam da reação de Maillard, juntamente com aminoácidos livres. A reformulação de produtos lácteos no sentido de diminuir o teor de lactose é realizada com o fim de tornar seu consumo adequado por indivíduos incapazes de metabolizar este açúcar, presente no leite entre 4\% e 7\% (Trani et al., 2017; Troise et al., 2016a). A diminuição do teor de lactose em cremes de leite UHT é frequentemente realizada pela indústria de lácteos através da hidrólise catalisada pela enzima lactase ( $\beta$-D-galactosidase), cuja adição pode ocorrer antes ou depois do tratamento térmico UHT (Troise et al., 2016b). Caso a adição de lactase seja feita após o tratamento térmico, ela se manterá viável e ativa durante o tempo de vida de prateleira. Troise et al. (2016a) encontraram maior quantidade de produtos da reação de Maillard em leites sem lactose cuja adição de lactase aconteceu após o tratamento térmico UHT, em comparação com aqueles em que a enzima foi adicionada após o tratamento térmico. Os autores atribuem isso ao efeito proteolítico que as misturas enzimáticas podem exercer durante o armazenamento, caso não apresentem elevado grau de pureza.

A avaliação sensorial é essencial para que as indústrias de alimentos conheçam as atitudes e percepções dos consumidores sobre seus produtos, o que pode garantir o sucesso ou não destes no mercado (Sidel \& Stone, 1993). As técnicas descritivas estão entre as ferramentas mais sofisticadas de análise sensorial, dentre as quais se destaca a Análise Descritiva Quantitativa (ADQ), que proporciona uma descrição completa de todas as propriedades sensoriais de um produto, bem como a intensidade em que ocorrem (Stone et al., 2012). Quando combinada com testes afetivos, fornece informações do impacto da intensidade de cada termo descritor sobre a percepção dos consumidores de determinada amostra, que pode ser positivo ou negativo (Lawless \& Heymann, 2010; Tenenhaus et al., 2005).

Nos últimos anos, surgiram no mercado brasileiro diversos tipos e marcas de creme de leite em embalagens longa vida, alguns com teor de gordura reduzido, outros livres de lactose. Independentemente da natureza da modificação, os consumidores desses produtos esperam que não haja prejuízo às suas características sensoriais, as quais contribuem para sua aceitação. Diante desse cenário, este estudo buscou identificar o perfil sensorial de seis amostras comerciais de creme de leite UHT e comparar os resultados com a aceitabilidade das amostras e a atitude de compra pelos consumidores, bem como com resultados de análises instrumentais de cor e textura, a fim de verificar quais características sensoriais deste produto definem maior ou menor aceitabilidade por parte dos consumidores.

\section{Material e métodos}

Seis amostras de $200 \mathrm{~g}$ de creme de leite homogeneizado $U H T$, embaladas em caixinhas longa vida, foram adquiridas em um supermercado na cidade de Campinas, SP, Brasil. O teor de gordura informado no rótulo das amostras 2, 4, 5 e 6 era $20 \%$, e das amostras 1 e 3 era 17\%, sendo que a amostra 1 era declarada como creme de leite leve no rótulo. As amostras 3 e 6 são do mesmo fabricante, sendo a amostra 6 descrita como $0 \%$ lactose na embalagem.

\subsection{Análise sensorial}

As sessões de análise sensorial foram realizadas no Laboratório de Ciência Sensorial e Estudos do Consumidor do Departamento de Alimentos e Nutrição da Faculdade de Engenharia de Alimentos (FEA - UNICAMP), em cabines com temperatura controlada $\left(20^{\circ} \mathrm{C}\right.$ a $\left.22^{\circ} \mathrm{C}\right)$ e luz branca. As amostras foram apresentadas aos provadores entre $22^{\circ} \mathrm{C}$ e $25^{\circ} \mathrm{C}$ em copos descartáveis de $50 \mathrm{~mL}$, de maneira monádica, e foi solicitado a eles que consumissem biscoitos tipo cream-cracker e água morna (a aproximadamente $50{ }^{\circ} \mathrm{C}$ ) entre cada amostra, para minimizar a influência da anterior sobre a seguinte. 


\subsection{Determinação do perfil sensorial}

O perfil sensorial das amostras de creme de leite foi determinado através da Análise Descritiva Quantitativa (ADQ), realizada segundo metodologia proposta por Stone et al. (2012). Treze alunos do Departamento de Alimentos e Nutrição da UNICAMP, que já tinham participado de outros testes sensoriais antes, foram pré-selecionados de acordo com sua disponibilidade, interesse e ausência de alergia ou intolerância ao leite e seus derivados.

O desenvolvimento da terminologia descritiva foi realizado pelo método de rede de Kelly (Moskowitz, 1983). Os provadores avaliaram as amostras em pares e apontaram suas diferenças e semelhanças de aparência, aroma, sabor e textura. Os termos gerados foram discutidos em reunião, e antônimos, sinônimos, termos redundantes e irrelevantes foram eliminados com o consentimento de todos os provadores. Os 22 termos descritores, para os quais foram determinadas definições e referências de intensidade mínima e máxima, estão listados na ficha da Tabela 1, utilizada como guia para o treinamento, o qual foi realizado em 4 sessões de uma hora.

Tabela 1. Termos descritores, suas definições e referências de intensidade máxima e mínima.

\begin{tabular}{|c|c|c|}
\hline $\begin{array}{c}\text { Termo } \\
\text { descritor }\end{array}$ & Definição & Referências \\
\hline \multicolumn{3}{|c|}{ Aparência } \\
\hline Cor creme & $\begin{array}{c}\text { Cor creme clara característica de creme de } \\
\text { leite }\end{array}$ & $\begin{array}{c}\text { Pouco: leite integral (Paulista }{ }^{\circledR} \text { ) } \\
\text { Muito: } 100 \mathrm{~mL} \text { de leite desnatado }\left(\text { Paulista }^{\circledR}\right) \text { com } \\
0,1 \mathrm{~g} \text { de café solúvel original }\left(\text { Nescafe }^{\circledR}\right)\end{array}$ \\
\hline $\begin{array}{l}\text { Viscosidade } \\
\text { aparente }\end{array}$ & $\begin{array}{l}\text { Facilidade da amostra em escorrer pelo } \\
\text { recipiente }\end{array}$ & $\begin{array}{l}\text { Pouco: iogurte batido sabor baunilha }\left(\text { Danone }^{\circledR}\right) \\
\text { Muito: iogurte grego }\left(\text { Vigor }^{\mathbb{\circledR}}\right)\end{array}$ \\
\hline Consistência & $\begin{array}{l}\text { Escoamento do creme ao ser suspenso com } \\
\text { colher e devolvido ao copo }\end{array}$ & 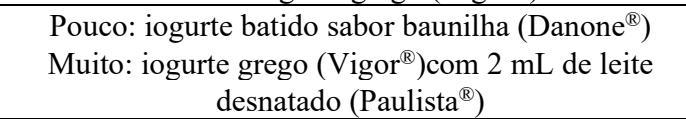 \\
\hline Homogeneidade & $\begin{array}{l}\text { Capacidade do creme de leite manter sua } \\
\text { aparência e estrutura em toda sua extensão, } \\
\text { sem grumos e/ou grânulos }\end{array}$ & $\begin{array}{l}\text { Pouco: } 10 \mathrm{~g} \text { de ricota (Queijos da Fazenda } \mathrm{JB}^{\circledR} \text { ) } \\
\text { com } 30 \mathrm{~g} \text { de creme de leite }\left(\text { Nestlé }^{\circledR}\right) \\
\left.\text { Muito: } 100 \mathrm{~g} \text { de requeijão (Vigor }{ }^{\circledR}\right) \\
\end{array}$ \\
\hline Aeração & Presença de bolha de ar no creme de leite & $\begin{array}{l}\left.\text { Nenhum: leite desnatado (Paulista }{ }^{\circledR}\right) \\
\text { Forte: álcool em gel }\left(\text { Zupp }^{\circledR}\right)\end{array}$ \\
\hline Brilho & Habilidade do creme de leite em refletir a luz & $\begin{array}{c}\text { Pouco: sobremesa láctea sabor chocolate branco } \\
\left.\text { (Danette, Danone }{ }^{\circledR}\right) \\
\text { Muito: leite integral }\left(\text { Paulista }^{\circledR}\right)\end{array}$ \\
\hline \multicolumn{3}{|c|}{ Aroma } \\
\hline Leite & Aroma característico de leite & $\begin{array}{l}\text { Pouco: leite desnatado (Paulista }{ }^{\mathbb{}} \text { ) } \\
\text { Muito: leite integral (Leitíssimo }{ }^{\circledR} \text { ) }\end{array}$ \\
\hline Creme de leite & Aroma característico de creme de leite fresco & $\begin{array}{c}\text { Pouco: } 50 \mathrm{~mL} \text { de creme de leite diluído }\left(\text { Nestlé }^{\circledR}\right) \text { em } \\
100 \mathrm{~mL} \text { de leite integral }\left(\text { Paulista }^{\circledR}\right) \\
\text { Muito: creme de leite fresco }\left(\text { Tirolez }^{\mathbb{Q}}\right)\end{array}$ \\
\hline Manteiga & Aroma característico de manteiga & $\begin{array}{l}\text { Nenhum: leite desnatado (Paulista }{ }^{\circledR} \text { ) } \\
\text { Forte: manteiga com sal }\left(\text { Aviação }^{\circledR} \text { ) }\right.\end{array}$ \\
\hline Requeijão & Aroma característico de requeijão & $\begin{array}{c}\text { Nenhum: leite desnatado }\left(\text { Paulista }^{\mathbb{B}}\right) \\
\text { Forte: requeijão }\left(\text { Vigor }^{\mathbb{R}}\right)\end{array}$ \\
\hline Leite em pó & Aroma característico de leite em pó & $\begin{array}{c}\text { Nenhum: leite desnatado } \\
\left.\text { Forte: } 2 \mathrm{~g} \text { de leite em pó diluído (Ninho, Nestlé }{ }^{\circledR}\right) \mathrm{em} \\
50 \mathrm{~mL} \text { de leite integral }\left(\text { Paulista }^{\circledR}\right)\end{array}$ \\
\hline Doce & $\begin{array}{c}\text { Aroma doce relacionado à presença de } \\
\text { açúcares }\end{array}$ & 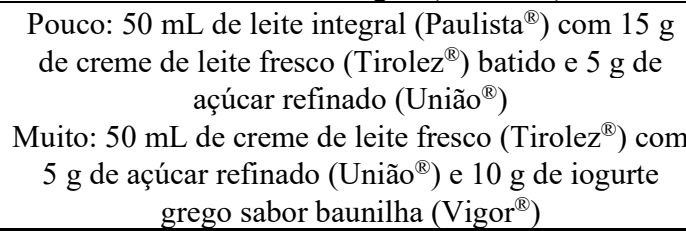 \\
\hline
\end{tabular}


Tabela 1. Continuação...

\begin{tabular}{|c|c|c|}
\hline $\begin{array}{c}\text { Termo } \\
\text { descritor }\end{array}$ & Definição & Referências \\
\hline \multicolumn{3}{|c|}{ Sabor } \\
\hline Leite & Sabor característico de leite integral & $\begin{array}{l}\text { Pouco: leite desnatado }\left(\text { Paulista }^{\mathbb{B}}\right) \\
\text { Muito: leite integral }\left(\text { Paulista }^{\mathbb{B}}\right)\end{array}$ \\
\hline Manteiga & Sabor característico de manteiga & $\begin{array}{c}\text { Nenhum: } 50 \mathrm{~mL} \text { leite desnatado }\left(\text { Paulista }^{\circledR}\right) \text { com } \\
50 \mathrm{~mL} \text { de água } \\
\text { Forte: manteiga sem sal }\left(\text { Aviação }^{\circledR}\right)\end{array}$ \\
\hline Doce & $\begin{array}{l}\text { Gosto doce relacionado à presença de } \\
\text { açúcares }\end{array}$ & $\begin{array}{c}\text { Nenhum: iogurte natural desnatado (Nestle }{ }^{\circledR} \text { ) } \\
\left.\text { Forte: } 50 \mathrm{~mL} \text { de iogurte batido (Danone }{ }^{\circledR}\right) \text { com } 20 \mathrm{~mL} \\
\text { de creme de leite (Nestle }{ }^{\circledR} \text { ) }\end{array}$ \\
\hline Leite em pó & Sabor característico de leite em pó & $\begin{array}{c}\text { Nenhum: leite desnatado (Paulista }{ }^{\circledR} \text { ) } \\
\text { Forte: } 50 \mathrm{~mL} \text { de leite integral }\left(\text { Paulista }{ }^{\circledR} \text { ) com } 2 \mathrm{~g} \text { de }\right. \\
\text { leite em pó (Ninho, Nestle }{ }^{\mathbb{B}} \text { ) }\end{array}$ \\
\hline Requeijão & Sabor característico de requeijão & $\begin{array}{c}\text { Nenhum: leite desnatado }\left(\text { Paulista }^{\circledR}\right) \\
\text { Forte: requeijão }\left(\text { Vigor }^{\mathbb{R}}\right) \\
\end{array}$ \\
\hline Nata & Sabor característico de nata & $\begin{array}{c}\text { Pouco: leite integral }(\text { Paulista } \\
\text { Muito: } 10 \mathrm{~mL} \text { de creme de leite fresco }\left(\text { Tirolez }^{\circledR}\right) \text { com } \\
50 \mathrm{~g} \text { de nata }\left(\text { Queijos da Fazenda } \mathrm{JB}^{\circledR} \text { ) }\right.\end{array}$ \\
\hline \multicolumn{3}{|c|}{ Textura } \\
\hline Corpo & Sensação de preenchimento na boca & $\begin{array}{l}\text { Pouco: iogurte batido }\left(\text { Danone }^{\mathbb{R}}\right) \\
\text { Muito: iogurte grego }\left(\text { Vigor }^{\mathbb{R}}\right)\end{array}$ \\
\hline $\begin{array}{c}\text { Textura } \\
\text { gordurosa }\end{array}$ & Sensação gordurosa durante a ingestão & $\begin{array}{c}\text { Pouco: leite integral }\left(\text { Paulista }^{\circledR}\right) \\
\text { Muito: manteiga com sal }\left(\text { Aviação }^{\circledR}\right)\end{array}$ \\
\hline $\begin{array}{l}\text { Residual } \\
\text { gorduroso }\end{array}$ & $\begin{array}{l}\text { Sensação gordurosa que permanece até } 15 \\
\text { segundos após a ingestão }\end{array}$ & $\begin{array}{c}\text { Pouco: leite integral }\left(\text { Paulista }^{\circledR}\right) \\
\text { Muito: nata (Queijos da Fazenda } \mathrm{JB}^{\circledR} \text { ) }\end{array}$ \\
\hline Fluidez & A facilidade do creme de leite de ser engolido & $\begin{array}{l}\text { Pouco: iogurte grego }\left(\text { Vigor }^{\mathbb{R}}\right) \\
\text { Muito: iogurte batido }\left(\text { Danone }^{\mathbb{R}}\right)\end{array}$ \\
\hline
\end{tabular}

A seleção dos provadores foi feita segundo sua capacidade discriminativa entre amostras, reprodutibilidade e concordância com o restante da equipe. Eles avaliaram a intensidade dos termos descritores de cada amostra em três repetições, utilizando uma ficha com escalas não estruturadas de $9 \mathrm{~cm}$, cujas extremidades esquerda e direita representavam, respectivamente, intensidades mínima e máxima. Os resultados foram submetidos à Análise de Variância (ANOVA) e foram selecionados os provadores que apresentaram probabilidade de $\mathrm{F}$ de amostra menor ou igual a 0,50 e probabilidade de $\mathrm{F}$ da repetição maior ou igual a 0,05 (Damasio \& Costell, 1991). Verificou-se o consenso das respostas de cada provador com o restante da equipe através da comparação das médias obtidas. Onze provadores treinados foram selecionados para determinação do perfil sensorial.

Os assessores avaliaram as amostras de acordo com as referências previamente determinadas para todos os termos descritores utilizando a ficha com as escalas. As amostras foram apresentadas sequencialmente de forma monádica em três repetições, em delineamento de blocos completos balanceados (MacFie et al., 1989). Os resultados foram tratados estatisticamente no software SAS 9.1.2 (SAS Institute, 2008), submetidos à ANOVA, provador e amostra como variáveis, e ao Teste de Médias de Tukey, com 5\% de significância.

\subsection{Testes com consumidores}

O teste de aceitabilidade foi realizado com 119 consumidores de produtos lácteos que não rejeitassem creme de leite, recrutados através de cartazes distribuídos na UNICAMP e redes sociais. Foi solicitado que provassem as amostras e indicassem o quanto gostaram da aparência, aroma, sabor, textura e aceitação global delass, utilizando uma escala hedônica linear não estruturada de $9 \mathrm{~cm}$ com âncoras de "desgostei extremamente", no extremo esquerdo da escala, e "gostei extremamente", no extremo direito da escala (Stone et al., 2012). Todas as amostras foram apresentadas de forma monádica, utilizando delineamento de blocos completos balanceados (Wakeling \& MacFie, 1995). Os consumidores também responderam a um 
questionário a respeito da sua intenção de compra em relação às amostras (Meilgaard et al., 1999). Utilizou-se uma escala de 5 pontos, em que 1 - certamente não compraria e 5 - certamente compraria.

Os resultados obtidos foram tratados estatisticamente por meio de ANOVA de dois fatores e Teste de Médias de Tukey, e foram correlacionados com os dados obtidos da ADQ através do método de Regressão Parcial dos Quadrados Mínimos. A impressão global do teste de aceitabilidade foi a variável dependente (matriz Y), enquanto os termos descritivos da ADQ foram as variáveis independentes (matriz X) (Cadena et al., 2013). Este tratamento foi realizado no software XLSTAT ao nível de significância de 5\%.

\subsection{Análises instrumentais}

As amostras foram submetidas às análises de cor e de viscosidade aparente para complementar as informações obtidas nas análises sensoriais. A determinação da cor foi realizada em um espectrofotômetro Colorquest II (Hunter-Lab ${ }^{\circledR}$ ), sistema de cor CIELAB, em que L* representa luminosidade, a* é o eixo vermelho-verde e b* o eixo amarelo-azul (Dufossé \& Galaup, 2009; Sousa et al., 2006). As amostras foram mantidas a temperatura ambiente até o momento da leitura, que foi realizada em 3 repetições por amostra.

Para determinação da viscosidade aparente, utilizou-se um reômetro programável digital BROOKFIELD $R V D V I I+$, com spindle $\mathrm{S} 15$ e relação de raios entre cilindros interno e externo de 0,75 . A temperatura foi mantida em 20,0 $\pm 0,5^{\circ} \mathrm{C}$ por meio de banho termostatizado BROOKFIELD TC500 durante as leituras. O modelo utilizado para cálculo da viscosidade aparente em função da rotação do spindle foi adaptado de Moura et al. (2001).

\section{Resultados e discussão}

\subsection{Análise descritiva quantitativa}

A Tabela 2 apresenta as médias obtidas no Teste de Médias de Tukey para os resultados da ADQ.

Tabela 2. Resultados do Teste de Médias de Tukey da ADQ das amostras de creme de leite.

\begin{tabular}{|c|c|c|c|c|c|c|c|}
\hline \multirow{2}{*}{ Cor creme } & 1 & 2 & 3 & 4 & 5 & 6 & MDS $^{2}$ \\
\hline & $2,89^{\mathrm{cd} 1}$ & $7,55^{\mathrm{a}}$ & $1,71^{\mathrm{e}}$ & $3,86^{c}$ & $4,9^{b}$ & $2,30^{\mathrm{de}}$ & 1,01 \\
\hline Viscosidade aparente & $2,56^{\mathrm{d}}$ & $3,79^{\mathrm{cd}}$ & $5,32^{\mathrm{ab}}$ & $4,22^{b c}$ & $6,08^{a}$ & $4,35^{b c}$ & 1,37 \\
\hline Consistência & $2,02^{\mathrm{d}}$ & $4,30^{\mathrm{bc}}$ & $5,58^{\mathrm{b}}$ & $4,15^{\mathrm{c}}$ & $7,11^{\mathrm{a}}$ & $4,80^{\mathrm{bc}}$ & 1,36 \\
\hline Homogeneidade & $7,65^{\mathrm{a}}$ & $7,58^{\mathrm{a}}$ & $5,69^{\mathrm{b}}$ & $4,27^{\mathrm{c}}$ & $7,45^{\mathrm{a}}$ & $5,53^{\mathrm{b}}$ & 1,09 \\
\hline Aeração & $1,54^{\mathrm{a}}$ & $0,80^{\mathrm{ab}}$ & $0,50^{\mathrm{b}}$ & $0,87^{\mathrm{ab}}$ & $0,47^{\mathrm{b}}$ & $0,65^{\mathrm{b}}$ & 0,78 \\
\hline Brilho & $6,32^{\mathrm{a}}$ & $6,45^{\mathrm{a}}$ & $6,28^{\mathrm{a}}$ & $5,73^{\mathrm{a}}$ & $5,91^{\mathrm{a}}$ & $6,05^{\mathrm{a}}$ & 0,75 \\
\hline Aroma de leite & $4,01^{\mathrm{d}}$ & $4,36^{\mathrm{cd}}$ & $6,47^{\mathrm{a}}$ & $5,28^{\mathrm{bc}}$ & $5,06^{\mathrm{bcd}}$ & $5,70^{\mathrm{ab}}$ & 1,18 \\
\hline Aroma creme de leite & $3,62^{\mathrm{b}}$ & $4,58^{\mathrm{ab}}$ & $5,13^{\mathrm{a}}$ & $5,23^{\mathrm{a}}$ & $5,23^{\mathrm{a}}$ & $4,33^{\mathrm{ab}}$ & 1,26 \\
\hline Aroma de manteiga & $1,17^{\mathrm{ab}}$ & $2,17^{\mathrm{ab}}$ & $1,12^{b}$ & $2,18^{\mathrm{a}}$ & $1,50^{\mathrm{ab}}$ & $1,14^{\mathrm{a}}$ & 1,05 \\
\hline Aroma de requeijão & $1,16^{\mathrm{a}}$ & $1,76^{\mathrm{a}}$ & $1,37^{\mathrm{a}}$ & $2,15^{\mathrm{a}}$ & $1,21^{\mathrm{a}}$ & $1,58^{\mathrm{a}}$ & 1,01 \\
\hline Aroma leite em pó & $1,90^{c}$ & $2,34^{\mathrm{b}}$ & $3,31^{\mathrm{a}}$ & $2,69^{\mathrm{ab}}$ & $2,90^{\mathrm{ab}}$ & $3,35^{\mathrm{a}}$ & 0,93 \\
\hline Aroma doce & $2,51^{\mathrm{a}}$ & $2,82^{\mathrm{a}}$ & $2,65^{\mathrm{a}}$ & $2,70^{\mathrm{a}}$ & $2,48^{\mathrm{a}}$ & $2,75^{\mathrm{a}}$ & 0,86 \\
\hline Sabor de leite & $5,37^{\mathrm{a}}$ & $3,62^{\mathrm{c}}$ & $5,27^{\mathrm{ab}}$ & $4,26^{\mathrm{bc}}$ & $4,36^{\mathrm{abc}}$ & $5,34^{\mathrm{ab}}$ & 1,11 \\
\hline Sabor de manteiga & $0,95^{\mathrm{d}}$ & $2,69^{a}$ & $1,76^{\text {bcd }}$ & $2,36^{\mathrm{ab}}$ & $1,95^{\mathrm{abc}}$ & $1,30^{\mathrm{cd}}$ & 0,85 \\
\hline Gosto doce & $3,19^{b}$ & $2,01^{\mathrm{c}}$ & $2,92^{\mathrm{bc}}$ & $2,51^{\mathrm{bc}}$ & $2,18^{\mathrm{bc}}$ & $5,39^{a}$ & 1,03 \\
\hline Sabor de leite em pó & $2,43^{\mathrm{ab}}$ & $1,75^{b}$ & $2,74^{\mathrm{ab}}$ & $2,09^{a b}$ & $2,24^{\mathrm{ab}}$ & $2,96^{\mathrm{a}}$ & 1,05 \\
\hline Sabor de requeijão & $0,90^{\mathrm{b}}$ & $1,96^{\mathrm{a}}$ & $1,70^{\mathrm{ab}}$ & $1,82^{\mathrm{ab}}$ & $2,02^{\mathrm{a}}$ & $1,55^{\mathrm{ab}}$ & 0,96 \\
\hline Sabor de nata & $2,01^{\mathrm{c}}$ & $3,54^{\mathrm{a}}$ & $2,55^{\mathrm{bc}}$ & $3,35^{\mathrm{ab}}$ & $2,68^{\mathrm{abc}}$ & $2,55^{\mathrm{bc}}$ & 0,89 \\
\hline Corpo & $2,32^{\mathrm{d}}$ & $3,98^{c}$ & $5,68^{\mathrm{ab}}$ & $3,82^{\mathrm{c}}$ & $6,72^{\mathrm{a}}$ & $4,75^{\mathrm{bc}}$ & 1,03 \\
\hline Textura gordurosa & $3,19^{c}$ & $5,17^{\mathrm{a}}$ & $4,39^{\mathrm{ab}}$ & $4,39^{\mathrm{ab}}$ & $4,79^{a}$ & $3,74^{\mathrm{bc}}$ & 1,04 \\
\hline Residual gorduroso & $2,92^{c}$ & $5,21^{\mathrm{a}}$ & $3,71^{b c}$ & $4,15^{\mathrm{ab}}$ & $4,91^{\mathrm{a}}$ & $3,68^{\mathrm{bc}}$ & 1,06 \\
\hline Fluidez & $6,37^{\mathrm{a}}$ & $4,91^{\mathrm{b}}$ & $3,37^{\mathrm{c}}$ & $5,04^{\mathrm{ab}}$ & $1,86^{\mathrm{d}}$ & $4,37^{\mathrm{bc}}$ & 1,39 \\
\hline
\end{tabular}

${ }^{1} \mathrm{Na}$ mesma linha, médias com letras em comum não diferem entre si ao nível de significância de $5 \%$. ${ }^{2} \mathrm{MDS}$ : mínima diferença significativa, obtida no Teste de Médias de Tukey $(p \leq 0,05)$. 
De maneira geral, as amostras variaram na maioria dos termos descritores, exceto brilho, aroma de requeijão e aroma doce, nos quais não houve discriminação entre as amostras ao nível de significância de $5 \%$. Em relação à cor creme, houve variação entre as amostras, sendo que a amostra 2 apresentou cor creme mais intensa que as demais, seguida das amostras 5 e 4 . A formação de compostos de coloração escura em produtos lácteos, como leite e creme de leite, pode estar relacionada com as altas temperaturas atingidas durante o tratamento térmico UHT. No entanto, variações nas médias de cor creme das amostras podem estar relacionadas à geração de produtos de coloração escura através da reação de Maillard durante o armazenamento, caso os produtos sejam expostos a ciclos de temperatura na vida de prateleira, conforme observado por Sunds et al. (2018).

Os provadores consideraram a amostra 6, zero lactose, como a mais doce. Este resultado era esperado pelos pesquisadores, pois a hidrólise da lactose gera glicose e galactose, compostos com dulçor relativo superior ao dissacarídeo original, contribuindo para maior percepção de dulçor nesta amostra (Adhikari et al., 2010; Monti et al., 2017; Moskowitz, 1970).

As amostras também foram discriminadas em relação aos termos descritores que caracterizam os aspectos reológicos do creme de leite, que são viscosidade aparente, consistência, fluidez e corpo. A amostra 5 foi caracterizada como a mais consistente, com maior viscosidade aparente, mais corpo e menos fluida $(p>0,05)$, seguida pelas amostras 3 e 6 . A amostra 1 foi considerada a menos consistente, com menor viscosidade aparente, menos corpo e mais fluida.

\subsection{Teste de aceitabilidade}

A Tabela 3 traz as médias da análise de aceitabilidade. As maiores diferenças entre as amostras foram percebidas em relação à aparência e textura. A amostra 5 teve as maiores médias de aparência e de textura, e não apresentou diferença significativa da amostra 6 para textura. A amostra 1 teve a menor média para textura, e a amostra 4 apresentou a menor média de aparência. Quanto ao aroma, sabor e impressão global, chama atenção o fato de que as amostras foram segmentadas em dois grupos pelos consumidores, sendo que as amostras 3, 5 e 6 não apresentaram diferença significativa entre si e receberam as maiores médias, e as amostras 1, 2 e 4 também não diferiram significativamente entre si e tiveram as menores médias.

Tabela 3. Resultados do Teste de Médias de Tukey do teste de aceitação de creme de leite.

\begin{tabular}{ccccccccc}
\hline Parâmetros de aceitabilidade & $\mathbf{1}$ & $\mathbf{2}$ & $\mathbf{3}$ & $\mathbf{4}$ & $\mathbf{5}$ & $\mathbf{6}$ & $\mathbf{M D S}^{\mathbf{2}}$ \\
\hline Aparência & $5,06^{\mathrm{c} 1}$ & $4,60^{\mathrm{c}}$ & $6,07^{\mathrm{b}}$ & $3,87^{\mathrm{d}}$ & $7,17^{\mathrm{a}}$ & $6,21^{\mathrm{b}}$ & 0,72 \\
\hline Aroma & $4,96^{\mathrm{b}}$ & $5,22^{\mathrm{b}}$ & $6,26^{\mathrm{a}}$ & $4,95^{\mathrm{b}}$ & $6,02^{\mathrm{a}}$ & $6,31^{\mathrm{a}}$ & 0,62 \\
\hline Sabor & $4,68^{\mathrm{b}}$ & $4,64^{\mathrm{b}}$ & $6,22^{\mathrm{a}}$ & $4,75^{\mathrm{b}}$ & $5,93^{\mathrm{a}}$ & $6,48^{\mathrm{a}}$ & 0,76 \\
\hline Textura & $3,71^{\mathrm{d}}$ & $4,92^{\mathrm{c}}$ & $6,07^{\mathrm{b}}$ & $4,14^{\mathrm{d}}$ & $6,97^{\mathrm{a}}$ & $6,25^{\mathrm{ab}}$ & 0,76 \\
\hline Impressão global & $4,50^{\mathrm{b}}$ & $4,91^{\mathrm{b}}$ & $6,26^{\mathrm{a}}$ & $4,47^{\mathrm{b}}$ & $6,50^{\mathrm{a}}$ & $6,49^{\mathrm{a}}$ & 0,67 \\
\hline
\end{tabular}

${ }^{1} \mathrm{Na}$ mesma linha, médias com letras em comum não diferem entre si ao nível de significância de $5 \%$. ${ }^{2} \mathrm{MDS}$ : mínima diferença significativa, obtida no Teste de Médias de Tukey $(p \leq 0,05)$.

A Figura 1 apresenta os dados da análise de intenção de compra. Pode-se observar que a tendência de os consumidores apresentarem maior intenção de compra para as amostras 3, 5 e 6 e menor intenção de compra para as amostra 1,2 e 4 está de acordo com as médias da análise de aceitabilidade. 


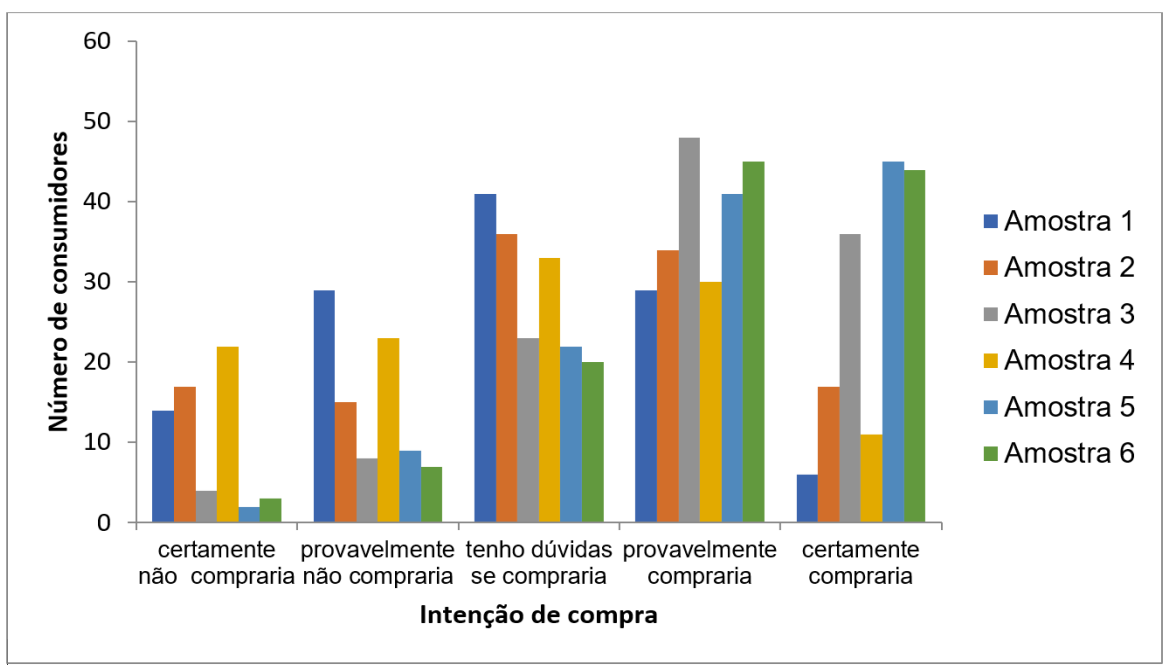

Figura 1. Intenção de compra das amostras de creme de leite.

\subsection{Análises instrumentais}

A Figura 2 mostra os valores dois eixos L* (luminosidade), a* (verde-vermelho) e b* (azul-amarelo) para as amostras de creme de leite. É possível observar que a amostra 2, à qual foi atribuída a maior média de cor creme pela equipe treinada, apresentou o componentes $L^{*}$ mais baixo, sendo a mais escura, e o componente $a^{*}$ mais elevado, ou seja, a mais avermelhada. Os valores de b* não variaram entre as amostras.

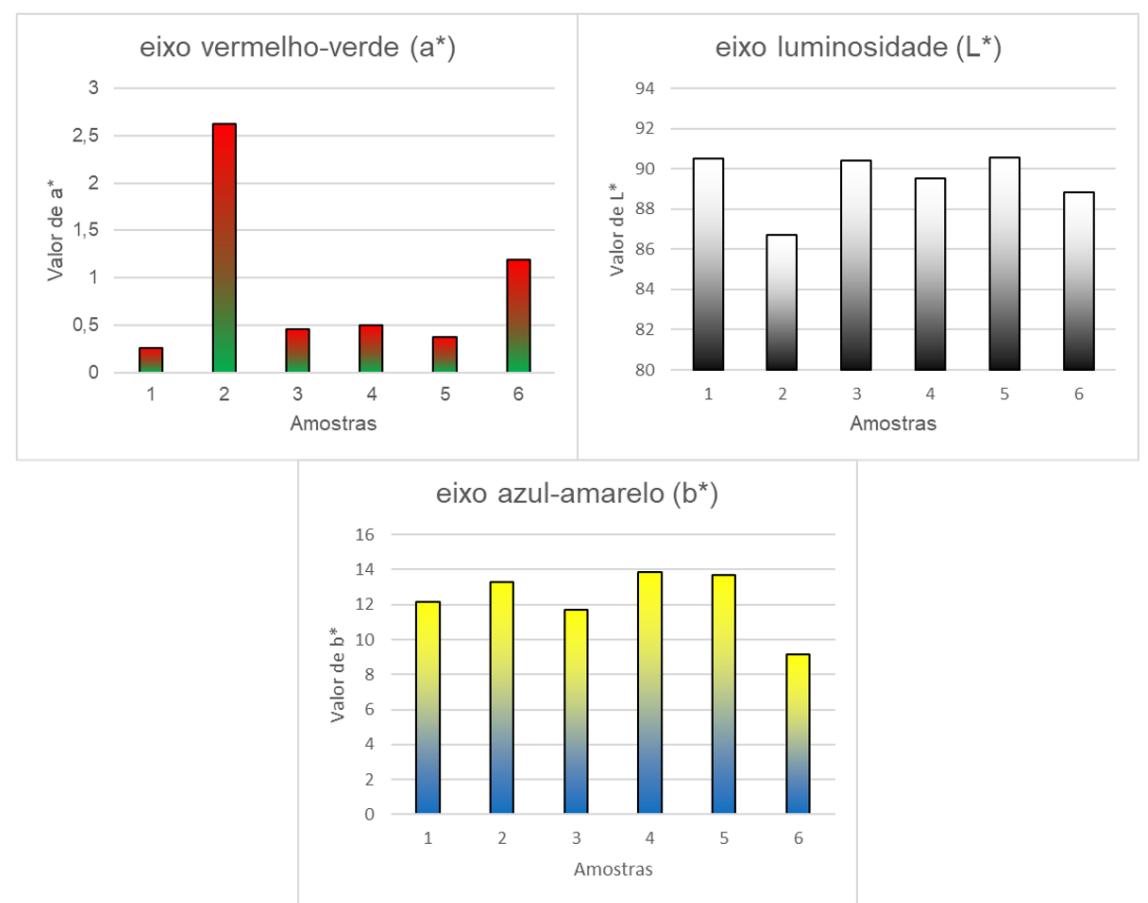

Figura 2. Resultados dos componentes $\mathrm{a}^{*} \mathrm{e} \mathrm{L}^{*}$ da análise de cor das amostras.

As amostras apresentaram comportamento reológico tixotrópico (Figura 3), já que os valores de viscosidade aparente diminuíram com o aumento da taxa de cisalhamento (Conti-Silva et al., 2018). Além disso, nota-se que a amostra 5 destaca-se em relação às outras amostras pelos seus valores de viscosidade aparente, que são muito superiores às demais. As amostras 1 e 2 apresentaram as menores viscosidades aparentes nas três rotações estudadas. 


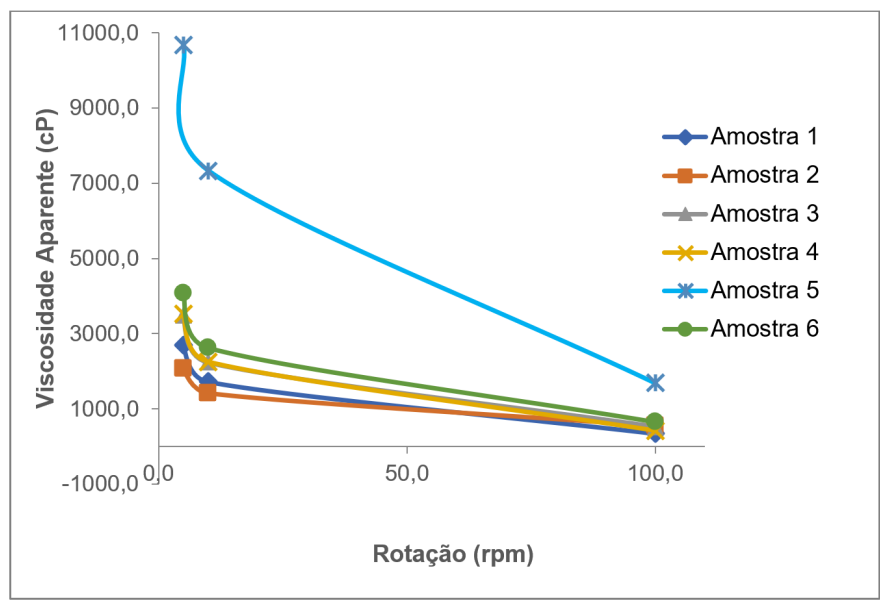

Figura 3. Viscosidade aparente de amostras comerciais de creme de leite UHT medida em reômetro nas rotações de 5,10 e $100 \mathrm{rpm}$ e expressas em cP.

\subsection{Regressão por quadrados mínimos parciais}

A análise de regressão por quadrados mínimos parciais correlaciona as médias obtidas na ADQ com as médias de impressão global do teste de aceitabilidade, a fim de identificar quais termos descritores aumentam (colunas azuis) ou diminuem (colunas vermelhas) a aceitabilidade das amostras pelos consumidores (Gomes et al., 2014).

Os resultados deste tratamento estatístico estão na Figura 4 e indicam que viscosidade aparente, consistência, aroma de leite em pó e corpo influenciaram de forma positiva a aceitabilidade das amostras, ao passo que fluidez, aroma de manteiga e aeração apresentaram efeito contrário. Além disso, foi observado que residual gorduroso e textura gordurosa não tiveram influência negativa sobre a aceitabilidade das amostras.

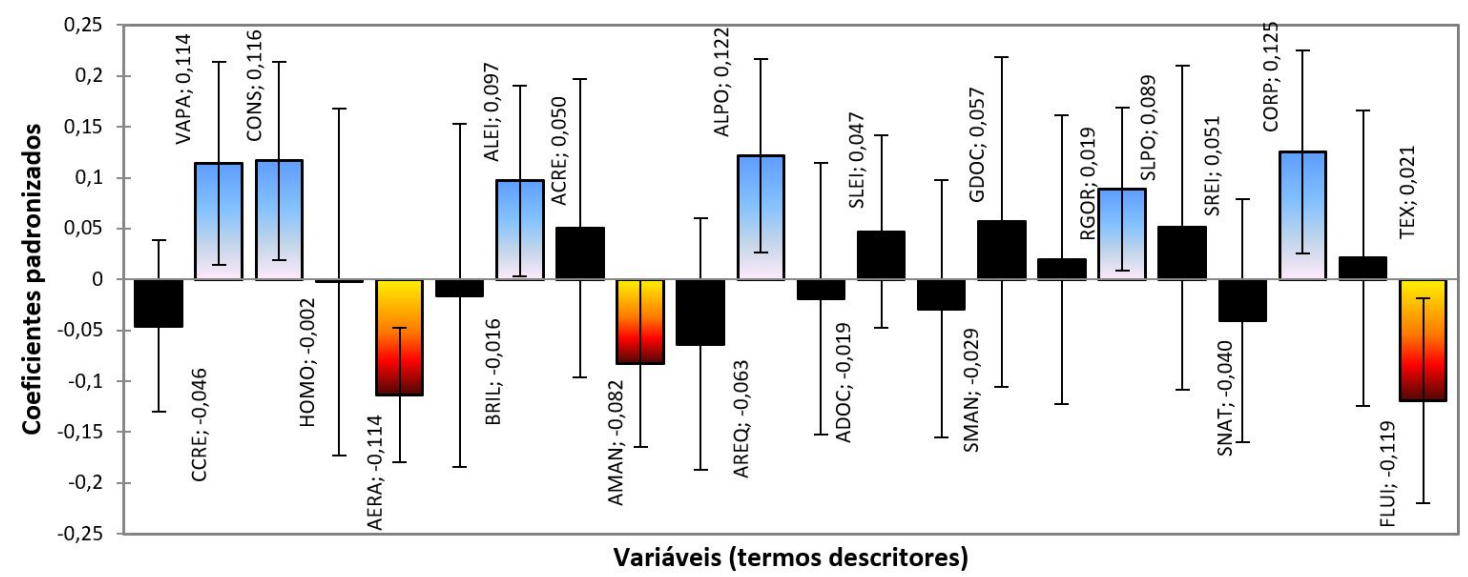

Figura 4. Análise de Regressão de Quadrados Mínimos Parciais. Colunas azuis = contribuição positiva para aceitabilidade; colunas vermelhas = contribuição negativa para aceitabilidade; colunas pretas = nenhuma contribuição para aceitabilidade; tamanho das colunas = intensidade do impacto sobre aceitabilidade (quando a linha vertical atravessa o eixo X, o termo correspondente não tem influência sobre aceitabilidade).

Os resultados sugerem que as propriedades reológicas dos cremes de leite homogeneizados UHT embalados em caixinha longa vida e comercializados no mercado brasileiro são determinantes para a aceitabilidade pelos consumidores e influenciam sua decisão de compra. Ainda que o termo cremosidade não 
tenha surgido durante a determinação do painel treinado, a preferência por cremes de leite mais viscosos, firmes e encorpados pelos consumidores pode estar associada com maior intensidade deste atributo sensorial.

Evidências sugerem que a viscosidade aparente é a propriedades física mais correlacionada com a percepção de cremosidade em emulsões lácteas semissólidas (Dickinson, 2018). No entanto, um estudo demonstra que, em produtos lácteos semissólidos, a alta viscosidade favorece a percepção de cremosidade enquanto o alimento se encontra na parte frontal da cavidade oral, e que, conforme o consumo progride, outras propriedades físicas do alimento determinam a percepção de cremosidade, como tamanho das partículas emulsionadas e fricção destas com a mucosa (Sonne et al., 2014). Além de possivelmente indicar maior cremosidade, a alta viscosidade de emulsões lácteas semissólidas também parece favorecer a expectativa de saciedade de consumidores, de maneira significativamente superior que o sabor (Hogenkamp et al., 2011).

A amostra 1, qualificada como a menos viscosa e mais fluida no painel sensorial e na análise instrumental, contém $17 \%$ de gordura, assim como a amostra 3 . No entanto, esta última não foi discriminada pelo painel treinado em relação ao corpo e à viscosidade aparente da amostra 5, a mais viscosa de todas, inclusive no teste instrumental. Portanto, não foi possível estabelecer uma relação direta da quantidade de gordura na amostra e sua viscosidade aparente, firmeza, corpo ou fluidez, reforçando a falta de correlação entre teor de gordura e parâmetros reológicos em produtos lácteos (Nguyen et al., 2016).

\section{Conclusão}

Ainda que o creme de leite homogeneizado UHT em caixinha longa vida seja um produto amplamente consumido no Brasil, não havia estudos que abordassem seus aspectos sensoriais até hoje. Este estudo é inovador, pois estabelece o perfil sensorial de variadas marcas e tipos deste produto, além de apontar quais são seus direcionadores de preferência. As informações obtidas serão úteis para profissionais de pesquisa e desenvolvimento na indústria de lácteos, no sentido de orientar a obtenção de produtos com maior aceitabilidade pelos consumidores.

\section{Referências}

Adhikari, K., Dooley, L. M., Chambers 4th, I. V. E., \& Bhumiratana, N. (2010). Sensory characteristics of commercial lactosefree milks manufactured in the United States. Lebensmittel-Wissenschaft + Technologie, 43(1), $113-118$. http://dx.doi.org/10.1016/j.Iwt.2009.06.017

Brasil. Ministério da Agricultura, Pecuária e Abastecimento. (1996, março 11). Aprova os Regulamentos Técnicos de Identidade e Qualidade dos Produtos Lácteos em anexo (Portaria $n^{\circ} 146$, de 7 de março de 1996). Diário Oficial da União, Brasília.

Cadena, R. S., Cruz, A. G., Netto, R. R., Castro, W. F., Faria, J. A. F., \& Bolini, H. M. A. (2013). Sensory profile and physicochemical characteristics of mango nectar sweetened with high intensity sweeteners throughout storage time. Food Research International, 54(2), 1670-1679. http://dx.doi.org/10.1016/j.foodres.2013.10.012

Conti-Silva, A. C., Ichiba, A. K. T., Silveira, A. L., Albano, K. M., \& Nicoletti, V. R. (2018). Viscosity of liquid and semisolid materials: Establishing correlations between instrumental analysis and sensory characteristics. Journal of Texture Studies, 49(6), 569-577. PMid:30156706. http://dx.doi.org/10.1111/jtxs. 12358

Damasio, M. H., \& Costell, E. (1991). Análisis sensorial descriptivo: Generación de descriptores y selección de catadores. Revista Agroquímica de Tecnologia de Alimentos, 31(2), 165-178.

Deosarkar, S. S., Khedkar, C. D., Kalyankar, S. D., \& Sarode, A. R. (2016). Cream: Types of cream. In B. Caballero, P. Finglas \& F. Toldrá (Eds.), Encyclopedia of food and health (Vol. 2, pp. 331-337). Oxford: Academic Press. http://dx.doi.org/10.1016/B978-0-12-384947-2.00205-1.

Dickinson, E. (2018). On the road to understanding and control of creaminess perception in food colloids. Food Hydrocolloids, 77, 372-385. http://dx.doi.org/10.1016/j.foodhyd.2017.10.014

Dufossé, L., \& Galaup, P. (2009). Color. In L. M. L. Nollet \& F. Toldrá (Eds.) Handbook of dairy foods analysis (1st ed., pp. 585605). Boca Raton: Taylor \& Francis Group.

Fox, P. F. (2011). Fat globules in milk. In J. W. Fuquay, P. F. Fox \& P. L. H. McSweeney (Eds.), Encyclopedia of dairy sciences (2nd ed., pp. 675-679). Oxford: Academic Press http://dx.doi.org/10.1016/B978-0-12-374407-4.00336-8.

Gallina, D. A., \& Antunes, A. E. C. (2017). Controle e adequação. In P. B. Zacarchenco, A. G. F. Van Dender \& R. A. Rego (Eds.), Brasil dairy trends 2020 (pp. 171-209). Campinas: Instituto de Tecnologia de Alimentos. 
Gomes, C. L., Pflanzer, S. B., Cruz, A. G., de Felício, P. E., \& Bolini, H. M. A. (2014). Sensory descriptive profiling and consumer preferences of beef strip loin steaks. Food Research International, 59, 76-84.

http://dx.doi.org/10.1016/j.foodres.2014.01.061

Gomes, R. A. R., Pithan e Silva, R. O., Van Dender, A. G. F., \& Zacarchenco, P. B. (2017). O setor de produtos lácteos. In P. B. Zacarchenco, A. G. F. Van Dender \& R. A. Rego (Eds.), Brasil dairy trends 2020 (pp. 11-46). Campinas: Instituto de Tecnologia de Alimentos.

Hoffmann, W. (2011a). Cream: Manufacture. In J. W. Fuquay, P. F. Fox \& P. L. H. McSweeney (Eds.), Encyclopedia of dairy sciences (2nd ed., pp. 675-679). Oxford: Academic Press. http://dx.doi.org/10.1016/B978-0-12-374407-4.00107-2.

Hoffmann, W. (2011b). Cream: Products. In J. W. Fuquay, P. F. Fox \& P. L. H. McSweeney (Eds.), Encyclopedia of dairy sciences (2nd ed., pp. 675-679). Oxford: Academic Press. http://dx.doi.org/10.1016/B978-0-12-374407-4.00108-4.

Hogenkamp, P. S., Stafleu, A., Mars, M., Brunstrom, J. M., \& de Graaf, C. (2011). Texture, not flavor, determines expected satiation of dairy products. Appetite, 57(3), 635-641. PMid:21871509. http://dx.doi.org/10.1016/j.appet.2011.08.008

Lawless, H. T., \& Heymann, H. (2010). Sensory evaluation of food: Principles and practices (2nd ed.). London: Springer. http://dx.doi.org/10.1007/978-1-4419-6488-5.

Lee, C. L., Liao, H. L., Lee, W. C., Hsu, C. K., Hsueh, F. C., Pan, J. Q., Chu, C. H., Wei, C. T., \& Chen, M. J. (2018). Standards and labeling of milk fat and spread products in different countries. Yao Wu Shi Pin Fen Xi, 26(2), 469-480. PMid:29567215. http://dx.doi.org/10.1016/j.jfda.2017.10.006

MacFie, H. J., Bratchell, N., Greenhoff, K., \& Vallis, L. V. (1989). Designs to balance the effect of order of presentation and firstorder carry-over effect in halls tests. Journal of Sensory Studies, 4(2), 129-148. http://dx.doi.org/10.1111/j.1745459X.1989.tb00463.x

Malmgren, B., Ardö, Y., Langton, M., Altskär, A., Bremer, M. G. E. G., Dejmek, P., \& Paulsson, M. (2017). Changes in proteins, physical stability and structure in directly heated UHT milk during storage at different temperatures. International Dairy Journal, 71, 60-75. http://dx.doi.org/10.1016/j.idairyj.2017.03.002

Meilgaard, M., Vance Civille, G., \& Thomas Carr, B. (1999). Sensory evaluation techniques (3rd ed.). Boca Raton: CRC Press. http://dx.doi.org/10.1201/9781439832271.

Monti, L., Negri, S., Meucci, A., Stroppa, A., Galli, A., \& Contarini, G. (2017). Lactose, galactose and glucose determination in naturally "lactose free" hard cheese: HPAEC-PAD method validation. Food Chemistry, 220, 18-24. PMid:27855887.

http://dx.doi.org/10.1016/j.foodchem.2016.09.185

Moskowitz, H. R. (1970). Ratio scales of sugar sweetness. Perception \& Psychophysics, 7(5), 315-320. http://dx.doi.org/10.3758/BF03210175

Moskowitz, H. R. (1983). Product testing and sensory evaluation of foods: Marketing and R\&D approaches. Westport: Food and Nutrition Press.

Moura, S. C. S. R., Vitali, A. A., \& França, V. C. L. (2001). Propriedades termofísicas de soluções modelo similares a creme de leite. Food Science and Technology, 21(2), 209-215. http://dx.doi.org/10.1590/S0101-20612001000200016

Nguyen, P. T. M., Bhandari, B., \& Prakash, S. (2016). Tribological method to measure lubricating properties of dairy products. Journal of Food Engineering, 168, 27-34. http://dx.doi.org/10.1016/j.jfoodeng.2015.07.011

SAS Institute. (2008). SAS user's guide: Statistics version 9.1.2. Cary: Sas Institute.

Sidel, J. L., \& Stone, H. (1993). The role of sensory evaluation in the food industry. Food Quality and Preference, 4(1-2), 65-73. http://dx.doi.org/10.1016/0950-3293(93)90314-V

Sonne, A., Busch-Stockfisch, M., Weiss, J., \& Hinrichs, J. (2014). Improved mapping of in-mouth creaminess of semi-solid dairy products by combining rheology, particle size, and tribology data. Lebensmittel-Wissenschaft + Technologie, 59(1), 342-347. http://dx.doi.org/10.1016/j.lwt.2014.05.047

Sousa, W. A., Pitombo, R. N. M., Silva, M. A. A. P., \& Marsaioli Junior, A. (2006). Sensory evaluation of dried bananas obtained from air dehydration assisted by microwaves. In M. Willert-Porada (Ed.), Advances in microwave and radio frequency processing (pp. 289-302). Berlin: Springer. http://dx.doi.org/10.1007/978-3-540-32944-2_31

Stone, H., Bleibaum, R. N., \& Thomas, H. A. (2012). Sensory evaluation practices. New York: Academic Press.

Sunds, A. V., Rauh, V. B., Sørensen, J., \& Larsen, L. B. (2018). Maillard reaction progress in UHT milk during storage at different temperature levels and cycles. International Dairy Journal, 77, 56-64. http://dx.doi.org/10.1016/j.idairyj.2017.08.008 Tenenhaus, M., Vinzi, V. E., Chatelin, Y. M., \& Lauro, C. (2005). PLS path modeling. Computational Statistics \& Data Analysis, 48(1), 159-205. http://dx.doi.org/10.1016/j.csda.2004.03.005

Trani, A., Gambacorta, G., Loizzo, P., Cassone, A., Fasciano, C., Zambrini, A. V., \& Faccia, M. (2017). Comparison of HPLC-RI, LC/MS-MS and enzymatic assays for the analysis of residual lactose in lactose-free milk. Food Chemistry, 233, 385-390. PMid:28530589. http://dx.doi.org/10.1016/j.foodchem.2017.04.134

Troise, A. D., Bandini, E., De Donno, R., Meijer, G., Trezzi, M., \& Fogliano, V. (2016a). The quality of low lactose milk is affected by the side proteolytic activity of the lactase used in the production process. Food Research International, 89(Pt 1), 514-525. PMid:28460946. http://dx.doi.org/10.1016/j.foodres.2016.08.021

Troise, A. D., Buonanno, M., Fiore, A., Monti, S. M., \& Fogliano, V. (2016b). Evolution of protein bound Maillard reaction endproducts and free Amadori compounds in low lactose milk in presence of fructosamine oxidase I. Food Chemistry, 212, 722729. PMid:27374589. http://dx.doi.org/10.1016/j.foodchem.2016.06.037 
Wakeling, I. N., \& MacFie, J. H. (1995). Designing consumer trials balanced for first and higher orders of carry-over effect when only a subset of k samples from t may be tested. Food Quality and Preference, 6(4), 299-308. http://dx.doi.org/10.1016/09503293(95)00032-1

Walstra, P., Wouters, J. T. M., \& Geurts, T. J. (2005). Cream products. In P. Walstra, J. T. M. Wouters \& T. J. Geurts (Eds.), Dairy science and technology (2nd ed., pp. 447-466). Boca Raton: CRC Press. http://dx.doi.org/10.1201/9781420028010.

Wilbey, R. A. (2011). Homogenization of milk: Principles and mechanism of homogenization, effects and assessment of efficiency: Valve homogenizers. In J. W. Fuquay, P. F. Fox \& P. L. H. McSweeney (Eds.), Encyclopedia of dairy sciences (2nd ed., pp. 750-754). Oxford: Academic Press. http://dx.doi.org/10.1016/B978-0-12-374407-4.00223-5.

Financiamento: Nenhum. 